\title{
Evaluación formativa, autorregulación, feedback y herramientas digitales: uso de Socrative en educación superior Formative assessment, self-regulation, feedback and digital tools: use of Socrative in higher education \\ *Juan Fraile, *Patricia Ruiz-Bravo, **David Zamorano-Sande, ***Daniel Orgaz-Rincón \\ *Universidad Francisco de Vitoria (España), **CES Don Bosco (España), ***Colegio Salesianos San Miguel Arcángel
} (España)

Resumen. Socrative es una herramienta digital de evaluación formativa que permite al docente crear itinerarios de aprendizaje a través de preguntas, introducir feedback que aparezca inmediatamente al responder y conocer el progreso de los estudiantes. En este artículo se expone una experiencia docente para la enseñanza de contenidos relacionados con las actividades físicas en el medio natural (AFMN) en el grado en Ciencias de la Actividad Física y del Deporte. Se fomentó el aprendizaje y la autorregulación a través de cuatro formas de feedback. Se recogieron datos sobre la percepción de la herramienta por parte de 30 estudiantes que la utilizaron en varias ocasiones a lo largo de la asignatura. Se empleó el cuestionario sobre la experiencia de buena práctica de la Red de Evaluación Formativa y Compartida en Educación (REFYCE), basado en el cuestionario validado sobre metodología y evaluación en formación inicial de profesorado (Castejón Oliva, Santos Pastor, \& Palacios Picos, 2015), junto a dos preguntas abiertas. Los resultados mostraron una gran satisfacción con esta actividad de aprendizaje, correlacionada con su valoración sobre la ayuda que recibieron de sus compañeros. Se presentan implicaciones pedagógicas relacionadas con la creación de grupos de alumnos para la realización de la actividad y sus distintos niveles, la dificultad, orden y naturaleza de las preguntas, el tipo de feedback introducido y el diseño de la tarea para un correcto andamiaje que fomente la autorregulación del aprendizaje.

Palabras clave: autoevaluación, coevaluación, corregulación, TIC, tecnología, competencias, universidad.

\begin{abstract}
Socrative is a formative assessment digital tool that allows the teacher to create learning itineraries through questions, introduce feedback that appears immediately when answering, and check the students' progress. This article presents a teaching experience for the instruction of contents related to physical activities in the natural environment in the degree in Physical Activity and Sport Sciences. Learning and self-regulation were encouraged through four forms of feedback. Data were collected on the perception of the tool by 30 students who used it on several occasions throughout the course. The questionnaire on good practice experience of the Spanish Network for Formative and Shared Evaluation in Education, based on the validated questionnaire on methodology and assessment in initial teacher training (Castejón Oliva et al., 2015), was used together with two open questions. The results showed high satisfaction with this learning activity, correlated with the appreciation of the assistance students received from their peers. Pedagogical implications are presented related to creating groups of students to carry out the activity and its different levels of performance, the difficulty, order and nature of the questions, the type of feedback introduced and the design of the task for a correct scaffolding that promotes self-regulated learning.
\end{abstract}

Keywords: self-assessment, peer-assessment, co-regulation, ICT, technology, competences, university.

\section{Introducción}

El grado en Ciencias de la Actividad Física y del Deporte (CCAFYDE) reúne una serie de perfiles profesionales que pueden tener los egresados determinados por la Agencia Nacional de Evaluación de la Calidad y Acreditación (ANECA, 2005): docencia en educación física (EF), entrenamiento deportivo, actividad física y salud, gestión deportiva y recreación deportiva. Con

Fecha recepción: 09-01-21. Fecha de aceptación: 23-05-21

Juan Fraile

juan.fraile@ufv.es importancia para el desempeño profesional en todos los perfiles, en el grado se imparten enseñanzas relacionadas con las Actividades Físicas en el Medio Natural (AFMN) (ANECA, 2005).

La regulación de la titulación de CCAFYDE viene indicada por el Real Decreto (RD) 1670/1993, de 24 de septiembre, donde se determinó qué debe incluir una asignatura específica de AFMN troncal, contando al menos con 4,5 créditos, y asegurándose de ese modo la inclusión obligatoria en todo el territorio nacional de al menos una materia relacionada con las AFMN. Esta asignatura se encuentra englobada dentro del área temática de Educación Física y Deportiva. Para consultar las últi- 
mas modificaciones, y de ese modo consultar las competencias del título, se debe acudir a la Resolución de 18 de septiembre de 2018, de la Secretaria General de Universidades por la que se publica el Acuerdo del Consejo de Universidades de 17 de septiembre de 2018, por el que se establecen recomendaciones para la propuesta por las universidades de memorias de verificación del título oficial de Grado en Ciencias de la Actividad Física y del Deporte en su apartado 4, planificación de la enseñanza, donde se presentan los módulos y créditos que deberá tener como mínimo el plan de estudios del grado en CCAFYDE. En el módulo 3, manifestaciones de motricidad humana, podemos encontrar, siendo de carácter obligatorio, entre sus descriptores el siguiente: «utilización didáctica de la actividad física en la naturaleza y en recursos naturales» (p. 91215). Por ello, las enseñanzas de AFMN son obligatorias dentro del plan de estudios del grado (y anterior licenciatura) en CCAFYDE.

\section{Las actividad físicas en el medio natural y la docencia en educación física}

En el marco de enseñanzas del grado en CCAFYDE, la adquisición de competencias relacionadas con las AFMN es importante para todos los perfiles y salidas profesionales mencionados anteriormente. Sin embargo, para el perfil relacionado con la docencia en educación física, será fundamental el dominio de estos contenidos ya que deberá transmitirlos en su actividad profesional de forma habitual, en cada curso académico.

Cabe destacar que es en el año 1990, con la aparición de la Ley orgánica de Ordenación General del Sistema Educativo (LOGSE, 1990), cuando se implantaron las AFMN como uno de los bloques de contenidos de la Educación Física. Con ello se le dio reconocimiento y valor a este tipo de prácticas en la formación del alumnado (Baena-Extremera, Palao Puche, \& Jara Cortina, 2015). Se asentaron de manera definitiva en el currículo de Educación Física gracias a la expansión que tuvo y los beneficios que se vio que aportaban al alumnado (Zabala, Dalmau, Viciana, \& Gargallo, 2003).

La implantación de dicho contenido en la ley educativa del momento provocó que los Institutos Nacionales de Educación Física (INEF) incluyeran dentro de sus planes de estudios la asignatura de AFMN, para la formación de sus estudiantes. Es a partir de entonces cuando las publicaciones de las AFMN cobran mayor importancia dado su carácter educativo y la necesidad que ello conlleva de innovar para el desarrollo de estos conteni- dos en las clases de Educación Física (Baena-Extremera et al., 2015).

Con el tiempo, se ha seguido manteniendo la aparición del contenido de AFMN en las diferentes leyes educativas. Con la Ley Orgánica de Educación (LOE, 2006), y más específicamente en su Real Decreto 1631/ 2006, de 29 de diciembre, por el que se establecen las enseñanzas mínimas correspondientes a la Educación Secundaria Obligatoria (2006, p. 60), se estableció en su bloque de contenidos 4, las Actividades en el Medio Natural. Se constituía como la oportunidad para que el alumnado interactuase de manera directa con el entorno que les es conocido y en el que se desarrollarían las actividades físicas. De ese modo, valorase su conservación mediante actividades sencillas y seguras. Con la Ley Orgánica para la Mejora de la Calidad Educativa (LOMCE, 2013) y de manera más específica en su Real Decreto 1105/2014, de 26 de diciembre, por el que se establece el currículo básico de la Educación Secundaria Obligatoria y Bachillerato (2014) en el que no se trabaja mediante bloques de contenidos, sino de situaciones motrices. Dentro de ellas, se encuentran las acciones motrices en situaciones de adaptación al entorno (p. 481) donde lo más significativo es que el medio en el que se realizan las actividades no tiene siempre las mismas características. Se hace alusión a desplazamientos con o sin material en entornos naturales o urbanos, pudiendo o no estar condicionados. Es importante la interpretación de las condiciones del entorno para situarse, velar por la seguridad y regular la intensidad de esfuerzos en función de las posibilidades personales. Es por ello por lo que las actividades que desde el marco normativo se recomienda realizar con el alumnado de secundaria y bachillerato son: marchas o excursiones a pie o en bicicleta, acampadas, actividades de orientación, etc. Estas modificaciones realizadas por la LOMCE han sido reformadas por la Ley Orgánica 3/2020, de 29 de diciembre, por la que se modifica la Ley Orgánica $2 /$ 2006, de 3 de mayo, de Educación (LOMLOE). Sin embargo, las modificaciones no implican cambios en los bloques de contenidos ni situaciones motrices que se abordan en esta investigación.

A pesar de esta estrecha relación entre el perfil profesional del docente de EF y las AFMN al ser un contenido obligatorio a impartir, Navarro, Arufe y Sancosmed (2015) determinaron que una gran parte del colectivo docente no desarrollaba los contenidos relacionados con las AFMN en sus clases. Son diversas las causas, siendo una de ellas la formación específica en dichas actividades y técnicas (Dalmau Torres, Jiménez Boraita, Gómez 
Estebas, \& Gargallo Ibort, 2020). Este hecho podría indicar también una deficiencia en la orientación y conocimientos del resto de egresados dedicados al resto de perfiles profesionales del grado.

Por tanto, una de las medidas está asociada a la mejora de los procesos de enseñanza-aprendizaje dentro de la formación inicial, universitaria, del alumnado para poder enfrentarse a dichas situaciones. Una buena formación inicial les permitirá afrontar con éxito las experiencias formativas referentes a las AFMN que tengan lugar tanto en la Educación Física como en el desempeño profesional asociado a otros perfiles. Así, en este artículo, se muestra cómo se ha desarrollado un proceso de evaluación formativa para la mejora del aprendizaje a través de Socrative, una herramienta digital.

\section{Justificación pedagógica}

\section{Evaluación formativa}

Los procesos de enseñanza-aprendizaje están profundamente ligados a la evaluación formativa (López Pastor, 2017). Este concepto de evaluación pretende la mejora del aprendizaje a través de una mayor implicación del alumnado en la identificación de puntos fuertes y débiles y su potenciación o corrección. La evaluación formativa no tiene como objetivo principal otorgar una calificación al alumnado, sino favorecer su aprendizaje y también el del docente, tomando conciencia de su práctica, para ser capaz de mejorarla (Pérez Pueyo, 2015). Entre las prácticas que componen la evaluación formativa se incluyen estrategias como compartir los criterios de evaluación con el alumnado, preguntar durante las clases, promover la producción y el intercambio de feedback, evaluar sin calificar, y fomentar la evaluación entre los estudiantes y la autoevaluación (Black \& Wiliam, 2009). Por desgracia, la autoevaluación y la evaluación entre pares se implementan poco, de acuerdo a lo explorado en las guías docentes (Lipnevich, Panadero, Gjicali, \& Fraile, 2021). Se debe tener en cuenta, tanto a la hora de leer este artículo como en relación a la literatura, que en castellano se suele emplear el término «coevaluación» mientras que en inglés sería "peerassessment», ya que la traducción más literal sería "coassessment» y esta se refiere a lo que se denomina en castellano «evaluación compartida» (López Pastor, 2017).

En conjunto, estas prácticas de evaluación formativa persiguen principalmente potenciar que los estudiantes sean capaces de autorregular su aprendizaje (Nicol \& Macfarlane-Dick, 2006). En una investigación en el grado en CCAFYDE, se observó que los estudiantes em- plean una gran variedad de estrategias al estudiar y realizar las tareas que se les solicitan, pero no todas son efectivas para conducirles al éxito académico ni son óptimas para que autorregulen su aprendizaje correctamente (García-Pérez, Fraile, \& Panadero, 2021). Para incentivar este proceso, los estudiantes deben conocer las metas marcadas, criterios o estándares con los que van a ser evaluados para, entre otros, compararlos con su trabajo a medida que lo van realizando e involucrarse así en el proceso de aprendizaje de forma activa (Panadero \& Romero, 2014). Estos estándares deben de estar accesibles al alumnado en todo el desarrollo de la tarea y no solo al final del proceso con el fin de iniciar el ciclo de autorregulación desde el inicio, con el planteamiento de metas (Panadero \& Romero, 2014). De esta forma, los estudiantes aprenderán siendo conocedores de qué se espera de ellos, mientras es acompañado por el docente -e incluso por sus compañeros-, que emitirán información sobre dónde se encuentra en su aprendizaje y cómo puede mejorar su desempeño. Estas premisas son válidas para cualquier tarea solicitada a los estudiantes a lo largo de la asignatura de forma independiente o bajo el paraguas de un portafolio que las compile y muestre el progreso, promoviendo el proceso de autorregulación (Fraile \& Pardo, 2016).

\section{Corregulación y feedback entre estudiantes para el aprendizaje}

El proceso cíclico de autorregulación está asociado a la sucesión de fases de feedback, consistentes en generar la información necesaria con la que se pueda reducir al máximo la distancia entre lo que se quiere lograr y el punto en el que se encuentra el estudiante (Black, Harrison, Lee, Marshall, \& Wiliam, 2003). Tradicionalmente, a través de las calificaciones finales, los estudiantes han recibido información muy tardía acerca de su rendimiento. De esa forma, ya no tenían posibilidad de emplearla y mejorar su desempeño. Por ello, los estudiantes deben ser partícipes en etapas previas con un papel activo en los ciclos de feedback (Winstone, Nash, Parker, \& Rowntree, 2017).

Este feedback no sólo ha de ser unidireccional, del docente al estudiante, sino que también puede -y debegenerarse a través de la autoevaluación y la coevaluación. Sin embargo, a pesar de la importancia de estos procesos, se ha visto por ejemplo que el fomento de la autoevaluación como estrategia docente por parte de los profesores del grado en CCAFYDE es escaso (Fraile, Pardo, \& Panadero, 2018).

En esta línea, se ha constatado que la interacción con 
sus compañeros y la intervención activa con los criterios de evaluación y el feedback mejora el aprendizaje y el proceso de autorregulación (Fraile, Gil-Izquierdo, Zamorano-Sande, \& Sánchez-Iglesias, 2020). Así, al compartir con sus iguales las interpretaciones de los criterios, generan una idea común de calidad a través del razonamiento (Topping, 2009). En estas estrategias de trabajo en parejas o grupo, los estudiantes se guían, ayudan, limitan o dan forma a actividades llevadas a cabo de forma colaborativa. Este proceso se denomina corregulación (Häkkinen et al., 2017). Con el fin de no confundir prácticas, conviene diferenciar la corregulación de la coevaluación. En esta última, los estudiantes evalúan y emiten juicios sobre el trabajo de otros compañeros (Topping, 2009), mientras que la corregulación es el proceso generado al realizar conjuntamente una tarea dos o más estudiantes -siendo el caso de la experiencia que narra este artículo-.

Estos procesos colaborativos que ejercitan la corregulación pueden ser apoyados a través de herramientas tecnológicas, lo que se denomina en inglés computer-supported collaborative learning (CSCL). Por tanto, el CSCL sería el soporte que permite abordar situaciones de trabajo en grupo a través del uso de tecnologías (Dillenbourg \& Fischer, 2007). En esta investigación se ha utilizado este apoyo tecnológico para crear un andamiaje en una tarea grupal donde se pretende fomentar la corregulación para la mejora del aprendizaje de cada estudiante (Järvelä \& Hadwin, 2013). El andamiaje es el diseño estructurado de la tarea a través de actividades o estrategias de apoyo que el profesor plantea para que el alumnado construya el conocimiento. En resumen, no se trata de ayudar al estudiante dándole las respuestas directamente, sino de diseñar un soporte estructurado para facilitar que alcance el objetivo de aprendizaje con su implicación activa (Kim, Belland, \& Walker, 2018). Investigaciones recientes han profundizado en los beneficios de la corregulación y la han relacionado con la autorregulación del aprendizaje (Panadero, Broadbent, Boud, \& Lodge, 2018). Estas investigaciones se basan en la idea de que a través de la regulación y el andamiaje que ofrece el docente, el CSCL o un compañero; el estudiante aprende a autorregularse mediante el modelo que le ofrecen estos agentes ( $\mathrm{Pa}-$ nadero et al., 2018). En el caso de esta experiencia, el CSCL y andamiaje creado se basa en la emisión automática de un feedback específico para cada uno de los problemas propuestos que el alumnado debía resolver en parejas. Para ello se empleó la herramienta Socrative, como se explica más adelante.

\section{Introducción a la propuesta y a la herra- mienta Socrative}

\section{Contenidos de aprendizaje}

Uno de los objetivos de esta asignatura de actividades físicas en el medio natural (AFMN) es aprender y poner en práctica los conceptos de cartografía y orientación. En esta experiencia se ha abordado la resolución de problemas relacionados con los contenidos de escala, rumbo, coordenadas geográficas y UTM, curvas de nivel, distancia y velocidad.

\section{Planteamiento didáctico de Socrative}

Socrative es una herramienta con muchas posibilidades para la evaluación formativa. Su uso se basa en la elaboración previa de preguntas con tres posibles tipos de respuesta: corta, múltiples o verdadero/falso. Posteriormente se lanza la actividad y los estudiantes únicamente tienen que emplear un dispositivo (ordenador, tableta o móvil) y el código que les proporciona el docente para acceder a la tarea, sin necesidad de loguearse.

\section{Tipo de actividad}

Hay dos tipos básicos de actividad: «prueba» o «quiz» y «carrera espacial» o «space race» (nombre en función del idioma en el que se tenga configurado Socrative. Inicialmente suele salir en inglés). Ambos modos de actividad son muy similares. La única diferencia es que «carrera espacial» tiene una connotación más competitiva y relacionada con la gamificación en la que cada alumno o equipo (cada dispositivo) tiene asociado un color y el docente puede proyectar una carrera que ganan aquellos que más preguntas acierten.

El modo empleado en esta experiencia es «prueba» o «quiz» debido a que el objetivo está en el aprendizaje exclusivamente y no se busca motivación extra a través de otros elementos de competición o gamificación. Otra razón importante a la hora de elegir esta modalidad es que en esta experiencia se han utilizado preguntas con respuestas cortas. Para conseguir que el sistema valide como correctas las respuestas cortas de los estudiantes, previamente tenemos que introducir las distintas posibilidades que, en caso de coincidir, devolvería como correcta la respuesta del estudiante. En este caso, al ser respuestas complejas en cuanto a su escritura y en las que se admite un pequeño margen de error de cálculo (por ejemplo, una coordenada como « $40^{\circ} 46^{\prime} 24^{\prime} \mathrm{N} 4^{\circ}$ 02' 57”W»), no tendría sentido condicionar la actividad a que se le diera por válida a los alumnos la respuesta introducida. Por ello, el sistema les puede trasladar que 
su respuesta es errónea y son los estudiantes los que tienen que considerar si es correcta por haberla escrito ligeramente diferente o teniendo en cuenta dicho margen de error -con las indicaciones previas del profesor en cuanto a dicha posible variación, por supuesto-.

A la hora de lanzar la actividad, existe la posibilidad de que les aparezcan las preguntas en un orden aleatorio y diferente para cada grupo. Esta característica se ha utilizado en ocasiones y es muy útil debido a que cada pareja de estudiantes aborda las mismas preguntas, pero en un orden distinto al de los compañeros, que provoca que se centren en la pregunta que les ha salido y deben resolver sin posibilidad de escuchar a los que estén cerca. Sin embargo, en otras ocasiones y como se redacta a continuación, hay problemas de cartografía que conllevan varias fases de resolución. En este caso, y con el fin de generar una estructura, progresión en dificultad y consolidación de los pasos que se han de dar, cada avance correspondía a una pregunta. Por tanto, no se podía activar el orden aleatorio de aparición.

\section{Uso de feedback}

En la misma línea, la gran característica de esta herramienta es la posibilidad de incluir una retroalimentación o feedback en cada pregunta (ver Figura 1). Normalmente, este está orientado a ayudar a los estudiantes cuya respuesta es incorrecta. Así, no necesitan acudir al profesor ante un error y se genera automáticamente una reflexión del alumnado apoyada en ese feedback que puede ayudar a comprender la razón de su fallo.

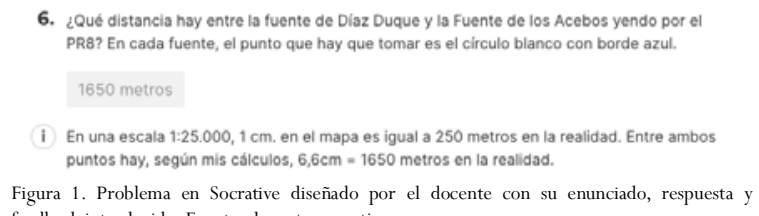

Es fundamental transmitir a los estudiantes que, ante un fallo, deben reflexionar sobre la razón que les ha llevado a cometerlo, para que se produzca un aprendizaje. En ocasiones, ver simplemente la respuesta correcta hará que se percaten e identifiquen el error; pero en muchas otras el feedback que aparecerá al responder -además de la respuesta correcta- generará esa autorregulación para reflexionar e identificar por qué habían fallado. Esto tiene un gran potencial porque los estudiantes tienen un papel activo al investigar las causas del error en vez de tener la intención de que el docente se lo explique. Además, el tiempo del profesor será mejor empleado ya que únicamente atenderá a los estudiantes que no han conseguido comprender la pregunta o el fallo que hayan cometido, a pesar del feedback automático recibido. Así, además, la explicación del profesor se apoya en reflexiones previas de los estudiantes que desempeñan un papel totalmente activo.

\section{Presentación de la propuesta didáctica}

Las actividades con Socrative en esta experiencia siempre se han llevado a cabo por parejas con el fin de favorecer la corregulación del aprendizaje entre los estudiantes gracias al debate entre ellos y la enseñanza de uno a otro. Además, se han tomado algunas decisiones en cuanto a las posibilidades que da la herramienta cuya elección se justifica en el punto anterior, con la introducción a la propuesta y a la herramienta Socrative. Este planteamiento didáctico se empleó a lo largo de tres sesiones para la adquisición de los contenidos de orientación y cartografía.

Cada tarea realizada constó de entre seis y diez problemas de respuesta abierta, cada uno con su feedback diseñado por el docente. La modalidad en Socrative al lanzar la actividad fue «prueba» o «quiz» ya que el objetivo primordial era el aprendizaje y no la motivación con elementos gamificados como en la modalidad «carrera espacial» o «space race». El orden de aparición de los problemas en ocasiones fue aleatorio y a veces fue el mismo para todas las parejas. Como se ha explicado anteriormente, la preferencia es la aleatoriedad, excepto si tuvo sentido que los problemas aparecieran en creciente dificultad para ir afianzando los conocimientos necesarios para los siguientes problemas más complejos. Tras la respuesta de los estudiantes, Socrative les indicaba si la respuesta era correcta y les mostraba el feedback que era la explicación de su resolución. Nuevamente, aquí se produce otro momento de autorregulación, en el que cada alumno reflexiona sobre su comprensión del resultado, de la comparación de la respuesta mostrada como correcta y de la suya. Asimismo, posteriormente, otro momento de corregulación, en el que los estudiantes entablan un debate para comprender el feedback, principalmente si han errado, y de explicación del uno al otro si un miembro no ha comprendido el problema y su solución. En esta línea, el docente les transmitió al inicio de la actividad que, si se equivocan en una pregunta, debían primero intentar identificar en qué habían fallado gracias al feedback que aparece. Otra instrucción que se les da es que, si no han entendido sus errores a pesar de haber 
leído el feedback, soliciten entonces ayuda al profesor. Es decir, el objetivo es proporcionar un feedback instantáneo tras emitir una respuesta y que los alumnos se hagan cargo de él, en un proceso activo y cognitivo de interacción con ese feedback. A continuación, si sigue habiendo dudas, pueden acudir al profesor, entablando un proceso dialógico que se apoya en la reflexión previa y compartida de los estudiantes. Así, en conclusión, se ha organizado y diseñado una tarea y un sistema de feedback automático que promueve la autonomía del alumnado y su implicación a través de procesos de autorregulación y corregulación y, además, se optimiza la participación del profesor a los momentos en los que de verdad es necesaria su intervención.

\section{Actividad transversal incluida en todas las se- siones}

En todas las ocasiones que se empleó Socrative se planteó al terminar la posibilidad de que los estudiantes crearan preguntas similares. El objetivo de ello fue doble. Por un lado, considerando que la creación de problemas parecidos, incluido el feedback, es otro proceso que mejora el aprendizaje de los estudiantes. Por otro lado, como estrategia docente para dar tiempo a las demás parejas a terminar la actividad y que todos los estudiantes tengan una tarea de aprendizaje que les ocupe. Socrative da la posibilidad de crear pruebas de elección múltiple mediante la importación de una plantilla en formato Excel. El archivo se puede descargar para ser cumplimentado y, posteriormente, importado. La idea fue reproducir este archivo Excel a partir de un Google Forms, ya que las respuestas de este último se trasladan también a un archivo Excel. Así, se podrían copiar y pegar en la plantilla e importar fácilmente las preguntas creadas por los estudiantes (ver videotutorial creado por el primer autor aquí: https://youtu.be/ aUOrX9WSjr8).

Este proceso fue también revisado por el docente con el fin de identificar que no hubiera fallos en esas preguntas creadas por los estudiantes por dos motivos. En primer lugar, para asegurarse de que no haya errores de concepto en el alumnado que lleve a cometer errores en la creación de los problemas. En segundo lugar, porque algunas de esas preguntas también fueron utilizadas posteriormente con todos los estudiantes.

\section{Método}

\section{Participantes y contexto}

En esta experiencia participaron 30 estudiantes que acudían regularmente a clase en la asignatura «Actividades deportivas en la naturaleza» del grado en Ciencias de la Actividad Física y del Deporte de una universidad de Madrid en el curso 2019-2020. El 53,3 \% fueron hombres. La edad media fue 21,2 años $(D T=3,01)$.

\section{Instrumento}

Para recoger la perspectiva de los estudiantes sobre las prácticas de evaluación formativa con el uso de la herramienta Socrative, se empleó un instrumento validado: la escala de percepción sobre metodologías participativas y evaluación formativa (EMPEF) (Castejón Oliva et al., 2015). Este instrumento valora la percepción del alumnado (y profesorado) de la evaluación formativa en la formación inicial en las titulaciones relacionadas con la educación física. De este cuestionario, para su análisis y presentación de resultados, se seleccionaron parte de los ítems ya que algunos de estos carecían de interés o sentido en el contexto de esta experiencia e investigación, siguiendo la línea del cuestionario sobre la experiencia de buena práctica de la Red de Evaluación Formativa y Compartida en Educación (REFYCE). El índice de fiabilidad de Cronbach fue de 0,6 (bajo, aunque lógico, por el bajo número de participantes; siendo de 0,84 en la validación original del cuestionario). Para garantizar la validez de las respuestas, los autores de este artículo decidieron incluir un ítem cuya redacción fue «Si lees esto, marca «poco» como respuesta. Se emplea para garantizar la validez de las respuestas (no lo comentes con otros participantes, por favor)». Dicho ítem se incluyó aproximadamente a la mitad del cuestionario y dentro de un bloque de cuatro ítems, ocupando el tercer lugar entre estos. Así, se emplearon los datos de 24 participantes (80 \%) que demostraron leer el cuestionario en el momento de responderlo, eliminando de la base de datos 6 respuestas $(20 \%)$.

Además, se añadieron dos preguntas abiertas consultando los aspectos positivos y negativos percibidos por los estudiantes en relación a la experiencia. Los estudiantes rellenaron el cuestionario de forma voluntaria a través de Google Forms.

\section{Análisis de datos}

Se realizó un análisis estadístico descriptivo cuantitativo complementado con un análisis cualitativo explicativo. En primer lugar, la investigación con los datos cuantitativos, mediante el software SPSS versión 22, a través de un análisis descriptivo de cada uno de los ítems del cuestionario. Además, se llevó a cabo un análisis de 
asociación de variables a través de la prueba de correlación de Spearman entre la variable considerada como principal (satisfacción global con la experiencia, medida a través de una variable cualitativa ordinal de cinco puntos) y otras variables consideradas como las más relevantes en el cuestionario: grado de dificultad de la experiencia, utilidad del aprendizaje adquirido, valoración de la ayuda recibida por el docente, y valoración de la ayuda recibida por los compañeros.

\section{Resultados}

\section{Resultados cuantitativos}

El análisis estadístico descriptivo mostró los resultados de las variables del cuestionario que se tuvieron en cuenta por el mayor interés en el contexto de esta experiencia (Tabla 1). Todas las variables presentaron valores muy altos. En especial, en una escala de cinco puntos (1-5) la satisfacción global de la experiencia $(M=$ $4,17, D T=0,7) \mathrm{y}$, principalmente, la valoración de la ayuda del docente $(M=4,71, D T=0,62)$. Asimismo, en una escala de cuatro puntos (1-4), los estudiantes otorgaron una puntuación muy alta a la utilidad de lo aprendido $(M=3,71, D T=0,55)$.

Además, la correlación de Spearman entre la variable satisfacción global de la experiencia y la valoración de la ayuda recibida por los compañeros resultó estadísticamente significativa $\left(r_{s}(24)=, 45, p<, 05\right)$; encontrando que se reporta una mayor satisfacción cuanto mejor se valoró la ayuda recibida por sus compañeros.

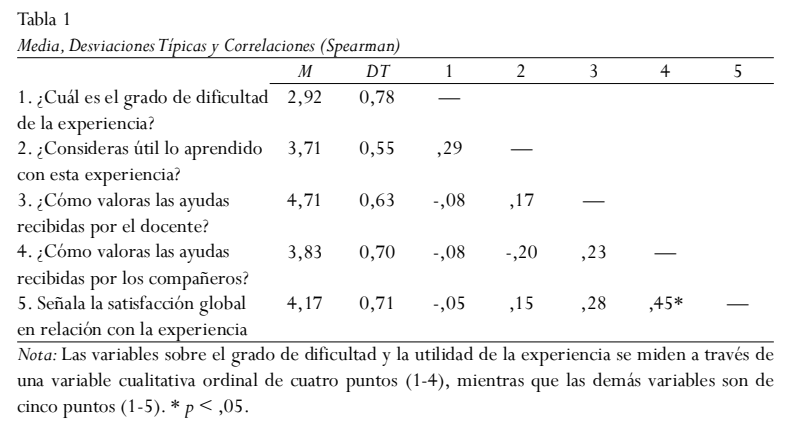

\section{Resultados cualitativos}

En cuanto al análisis de los datos cualitativos a partir de preguntas abiertas, como aspectos positivos los estudiantes destacan las posibilidades de la herramienta en cuanto a su utilidad, facilidad de uso o hacer más atractivo el aprendizaje. Varios estudiantes valoran el funcionamiento de la herramienta al permitirles ser autónomos en su aprendizaje y también por poder recibir feedback automáticamente. Aquí presentamos un extracto de la opinión de dos estudiantes:

Puedes trabajar por tu cuenta y la propia aplicación te da la respuesta y te explica el porqué y te da feedback.

\section{(Estudiante \#18)}

Me encanta responder y tener la pregunta corregida al momento y con una explicación de por qué está bien o mal mi respuesta, es muy útil para aprender.

(Estudiante \#20)

Además, consideran una ventaja el trabajo en parejas para su aprendizaje, como ilustra la siguiente frase:

Te permite trabajar con la ayuda de un compañero de cara a un ejercicio tipo examen, dos mentes trabajan mejor que una.

\section{(Estudiante \#9)}

En relación a los aspectos negativos, los estudiantes coinciden en destacar principalmente dos. En primer lugar, varios estudiantes comentaron que debían introducir la respuesta igual que la que había puesto el profesor como válida en Socrative para que esta fuera dada como correcta. Este primer aspecto negativo se explica y justifica en el siguiente apartado ampliamente. En segundo lugar, aquellos relacionados con el trabajo en parejas. Al contrario de los que veían en ello una ventaja, otros compañeros identifican problemas relacionados con su pareja, bien por su desinterés, por su bajo nivel y la inversión de tiempo y esfuerzo en explicarle todo o por las largas discusiones si continuamente estaban en desacuerdo:

En las situaciones de incertidumbre es fácil que se den los desacuerdos que deriven en frustración hacia la materia.

(Estudiante \#07)

Tuve el problema de que al final acabé haciéndolo todo solo.

(Estudiante \#11)

\section{Decisiones de acción}

\section{Socrative, ¿herramienta óptima?}

Hay multitud de herramientas digitales que tienen funciones parecidas (Kahoot, Quizizz, Plickers, Nearpod...) y, por ello, se debe escoger muy bien la ocasión en la que emplear cada una de ellas en función de sus características. Como se exponía anteriormente, y hablando en concreto de Socrative, el modo «prueba» empleado en esta experiencia no persigue en ningún caso la competición; mientras que el modo «carrera espacial» sí aporta el factor competitivo y gamificado. Siempre hay que considerar cuándo se debe emplear 
uno y otro y, para una actividad totalmente encaminada al aprendizaje, como la descrita en este artículo, recomendamos este modo «prueba». Podríamos comparar este aspecto con el uso de Kahoot, quizás la herramienta más conocida para realizar preguntas de respuesta múltiple en un ambiente competitivo. Una reciente revisión expone que, a pesar de sus muchas características positivas, un destacable aspecto negativo de Kahoot es que la puntuación no sólo se basa en acertar, sino en la velocidad con la que los estudiantes respondan (si es correcta, a más velocidad más puntos), reduciendo la reflexión y haciendo que algunos adivinen sin pensar (Wang \& Tahir, 2020). Evidentemente, el principal fin suele ser el aprendizaje, aunque en función de la herramienta y del uso que se haga de la misma, dicho objetivo puede verse mermado en función de otros como la motivación, cambiar el ritmo o el clima de la clase, etc. Como todo, la elección pasa por la necesidad detectada y el objetivo docente.

Considerando la finalidad de maximizar el aprendizaje, reducir al mínimo la importancia de la competición y programar un feedback automático; hemos identificado otras opciones que finalmente se descartaron. Dos de ellas, muy similares, son Google Forms y Microsoft Forms. El aspecto negativo es que el feedback lo reciben después de haber contestado todas las preguntas, por lo que no es tan inmediato como en Socrative y, por consiguiente, no enmienda los posibles errores de una pregunta a la siguiente. Aquí entra en juego otra variable: la versión de pago de Socrative. Personalmente, consideramos que no merece la pena su compra, aunque es verdad que la versión gratuita de Socrative sólo permite lanzar una prueba a la vez (se podrían lanzar varias con diferentes cuentas asociadas a distintas direcciones de email). La ventaja de poder lanzar actividades diferentes y a la vez reside en que se pueden atender distintos niveles en la clase, concatenar dos tareas seguidas en progresiva dificultad, los estudiantes puedan elegir qué tarea les conviene más, etc.

Por otro lado, Moodle también puede ser una gran elección porque dispone de muchísimas opciones, incluidas todas las que se han comentado. Su gran desventaja es la dificultad para el docente de aprender cómo manejar todas estas posibilidades, muy poco intuitivas, y el diseño visual en sí.

Definitivamente, para este tipo de tarea, nos quedamos con Socrative debido a su facilidad de uso para docente y estudiantes, feedback inmediato y posibilidad de respuestas abiertas.

\section{Tipo de preguntas: ¿abiertas o cerradas?}

Uno de los aspectos negativos señalado por algunos estudiantes, como se expone en el apartado de resultados, era que Socrative les daba por errónea una respuesta que habían escrito bien. El motivo de esto es que no la habían introducido exactamente como el docente había puesto la respuesta correcta. Es verdad que Socrative da la posibilidad de que el docente introduzca varias respuestas abiertas como verdaderas y, si los estudiantes escriben una de ellas, se la dará por válida. Sin embargo, siempre existirá la posibilidad de que los alumnos la escriban de alguna forma que sea incorrecta para el sistema, pero que el propio docente daría por buena. Asimismo, todo depende de la naturaleza de la pregunta ya que, como en esta experiencia, en respuestas como una coordenada ( $440^{\circ} 46^{\prime} 24^{\prime} \mathrm{N}^{\circ} 02^{\prime} 57^{\prime}$ ” $\left.\mathrm{W} »\right)$ puede ser difícil coincidir, además de que el docente permite un cierto margen de error.

La solución anterior no la consideramos como un punto de mejora de esta experiencia ya que, por un lado y como comentábamos, no tiene solución en todos los casos; y, por otro lado, el docente debe transmitir a los estudiantes que es una actividad de aprendizaje en la que no hay competición. Si los alumnos al comparar su respuesta introducida con la/s que salen como válidas identifican que estaba bien, entonces han de tomarlo como un acierto. A pesar de todo esto, es de destacar la importancia que los estudiantes dan a que Socrative les dé por buena la respuesta introducida, aunque ellos mismos ya vean que es así. Por ello, y acorde a los resultados, será importante insistir en que la actividad es exclusivamente de carácter formativo y orientada al aprendizaje exclusivamente, nada competitivo ni con una calificación asociada.

Sin embargo, hay un aspecto a considerar que afecta a la toma de decisiones del docente tras ver el avance y aprendizaje de sus alumnos. A la hora de que el profesor explore los aciertos y fallos de sus estudiantes de un vistazo en Socrative, uno de los problemas tiene que ver exclusivamente con la naturaleza de las preguntas abiertas. Si el profesor no explora qué respuestas dio Socrative por erróneas cuando debían ser válidas -como se ha explicado ampliamente en este subapartado- podría llevarle a error al considerar que algunos alumnos no han adquirido conocimientos por ligarlo a errores cometidos en Socrative.

\section{Dificultad y orden de las preguntas}

Otro aspecto fundamental que considerar es la dificultad y el orden de las preguntas. En cuanto al primer 
aspecto, si en la actividad hay unas preguntas más sencillas que otras, consideramos que el docente debería ordenarlas de menor a mayor dificultad y configurar la actividad de forma que a todos les salgan en el mismo orden. La razón principal es buscar que los conocimientos más básicos se vayan asentando y, finalmente, los estudiantes puedan responder a preguntas más complejas que les irán apareciendo según avanza la actividad.

En la introducción comentábamos que una característica útil es la aparición de las preguntas en un orden aleatorio para que los estudiantes no estén pendientes de otros grupos. Insistimos en que esto se debe hacer sólo cuando la dificultad de todas las preguntas sea la misma.

\section{Andamiaje y autorregulación del aprendizaje}

De cara a evaluar el itinerario de aprendizaje diseñado, consideramos fundamental reflexionar sobre las preguntas acertadas y erradas por los estudiantes -teniendo en cuenta la excepcionalidad expuesta anteriormente en cuanto al carácter de las preguntas abiertas-. Por un lado, tendrían utilidad para identificar en qué preguntas han errado más los estudiantes para luego explorar por qué ha sido. Quizás, por ejemplo, podría deberse a un error en el diseño de la pregunta o del feedback, de concepto por parte del alumno o del docente cuando lo explicó. Además, este error puede aclararse al hacer el ejercicio a través de Socrative, ver el feedback y también recibir una explicación del docente. Por ello, además de revisar de forma general los aciertos y fallos en cada pregunta, será muy útil recibir el feedback de los estudiantes sobre el diseño de las preguntas. Asimismo, también se podría, y debería, combinar con algún tipo de prueba o actividad individual para tener otra aproximación a los conocimientos de los estudiantes.

Otra tarea que nos parece fundamental y que fomenta el aprendizaje es la creación de preguntas similares. En el caso de esta experiencia, como se ha explicado y con un link a un videotutorial en YouTube, se hizo a través de un formulario de Google Forms para luego poder importarlas fácilmente y utilizarlas. Además, como se apuntaba, esta dinámica tiene otra función relacionada con ocupar a los estudiantes con otras tareas tras haber concluido la actividad con Socrative.

Grupos de trabajo e itinerario de aprendizaje: ¿prediseñados o a la elección de los estudiantes?

Considerando que los resultados mostraron la correlación entre la satisfacción con esta actividad de apren- dizaje y su valoración sobre la ayuda que recibieron de sus compañeros, una propuesta de mejora sería identificar con una prueba individual -o que ellos mismos digan qué nivel de competencia consideran que tienen-su nivel para emparejarles según este. Así, no sólo proporcionar ejercicios para distintos niveles o en base a sus debilidades, sino que el docente pueda estar más atento y acompañar mejor a los estudiantes que tienen menos pericia, a la vez que se proporciona una actividad que ponga a prueba a cada alumno de acuerdo a su nivel.

Otra opción sería el emparejamiento heterogéneo. Así, trabajarían juntos aquellos que hayan demostrado mayores conocimientos con los que menos destreza tengan, para así actuar con ellos como profesores.

\section{Conclusiones}

Esta experiencia docente e investigación, en un contexto de evaluación formativa, proporciona implicaciones prácticas y teóricas sobre la enseñanza de contenidos de Actividades Física en el Medio Natural (AFMN) a través del uso de tecnología mediante Socrative.

Cuatro formas de feedback se unen en esta tarea y han sido altamente valoradas por los estudiantes en relación a su aprendizaje. En primer lugar, por la autoevaluación y autorregulación que implica el rol con el que cada estudiante se aproxima a la tarea, cuando se ponen en juego sus conocimientos y habilidades con los problemas que tiene que resolver. En segundo lugar, dicha acción individual que conduce a la corregulación entre estudiantes con constantes verificaciones y ayudas entre ellos que se brindan a través del diálogo. De hecho, el principal resultado del estudio es que la satisfacción global de los estudiantes con esta actividad de aprendizaje se correlacionó con su valoración sobre la ayuda recibida de sus compañeros en la tarea. En tercer lugar, por el feedback automático y explicativo introducido por el docente en Socrative. Los alumnos lo obtienen inmediatamente, en el momento preciso, tras la resolución de cada problema. En cuarto y último lugar, y siguiendo el mismo orden temporal de la actividad propuesta en esta experiencia, el feedback del docente. Este sólo llega cuando realmente los estudiantes le necesitan porque, a pesar de la implicación cognitiva de los alumnos en todos los procesos anteriores, no lo han resuelto. Por ello, el diseño de esta tarea maximiza el rol del profesor al acudir donde y cuando realmente se le necesita. Igualmente, como se exponía ampliamente en el apartado anterior, «decisiones de acción», este punto implica una necesaria reflexión por parte del docente. 
El profesor tiene que preguntarse por qué los estudiantes han tenido que acudir finalmente a él/ella para saber qué ha fallado en todo el proceso previo organizado.

Consideramos que Socrative es una herramienta excelente para diseñar un itinerario de aprendizaje. Mediante preguntas, incluso de respuesta abierta -destacamos esto como una gran característica-, que los estudiantes responden a su propio ritmo, que pueden debatir en parejas y que permite al docente escribir un feedback que aparecerá tras la respuesta a cada pregunta. De esta forma, da la oportunidad al profesor de diseñar al detalle la secuencia de un itinerario o proceso de aprendizaje.

Como conclusión última, se aprecia que el diseño de esta tarea proporciona un andamiaje de enseñanza, evaluación formativa, autorregulación y corregulación. El uso de la tecnología, a través de Socrative, promueve que se den óptimamente varios procesos de feedback y regulación del aprendizaje a la vez, en los que los alumnos son verdaderos agentes activos y se optimiza el papel del profesor durante la actividad.

\section{Financiacion}

Este trabajo ha sido financiado por la Universidad Francisco de Vitoria en la Convocatoria de Investigación en Innovación Educativa 2020 en el proyecto «Empleo interdisciplinar y formativo de las rúbricas en educacion superior» (UFV2020-46).

\section{Referencias}

Agencia Nacional de Evaluación de la Calidad y Acreditación (ANECA). (2005). Libro Blanco delTítulo de Grado en Ciencias de la Actividad Física y del Deporte.

Baena-Extremera, A., Palao Puche, P., \& Jara Cortina, D. (2015). Evolución de las investigaciones sobre la enseñanza de las actividades en el medio natural en educación física escolar. Espiral. Cuadernos Del Profesorado, 8(16), 13 18. https: / / doi.org/10.25115/ecp.v8i16.984

Black, P., Harrison, C., Lee, C., Marshall, B., \&Wiliam, D. (2003). Assessment for learning: Putting it into practice. Maidenhead: Open University Press.

Black, P., \& Wiliam, D. (2009). Developing the theory of formative assessment. Educational Assessment Evaluation and Accountability, 21(1), 5-31.https://doi.org/10.1007/ s11092-008-9068-5

Castejón Oliva, F. J., Santos Pastor, M. L., \& Palacios Picos,A. (2015). Cuestionario sobre metodología y evaluación en formación inicial en educación física. Revista Internacional de Medicina y Ciencias de La Actividad Física y El Deporte,
15(58), 245-267. https://doi.org/10.15366/ rimcafd2015.58.004

DalmauTorres, J. M., Jiménez Boraita, R., Gómez Estebas, N., \& Gargallo Ibort, E. (2020). Diagnóstico escolar sobre el tratamiento de las actividades físicas en el medio natural dentro de la asignatura de Educación Física. Retos, 37, 460464. https://doi.org/10.47197/retos.v37i37.71010

Dillenbourg, P., \& Fischer, F. (2007). Computer-supported collaborative learning: The basics. Zeitschrift Für BerufsUndWirtschaftspädagogik, 21, 111-130.https://doi.org/ 10.1002/9781118766804.wbiect195

Fraile, J., Gil--zquierdo, M., Zamorano-Sande, D., \& SánchezIglesias, I. (2020). Self-regulated learning and formative assessment process on group work. Revista Electrónica de Investigación y Evaluación Educativa (RELIEVE), 26(1), M5. https://doi.org/10.7203/relieve.26.1.17402

Fraile, J., \& Pardo, R. (2016). El ePortafolio como instrumento para fomentar la autorregulación del aprendizaje. Revista Didáctica, Innovación y Multimedia, 34, 1-11.

Fraile, J., Pardo, R., \& Panadero, E. (2018).Autoevaluacion y autocalificacion en el grado en Ciencias de la Actividad Física y del Deporte: Estudio censal de las guías docentes. Profesorado. Revista de Curriculum y Formacion de Profesorado, 22(3), 163-182. https://doi.org/10.30827/ profesorado.v22i3.7997

García-Pérez, D., Fraile, J., \& Panadero, E. (2021). Learning strategies and self-regulation in context: how higher education students approach different courses, assessments, and challenges. European Journal of Psychology of Education, 36(2), 533-550. https://doi.org/10.1007/ s10212-020-00488-z

Häkkinen, P., Järvelä, S., Mäkitalo-Siegl, K., Ahonen, A., Näykki, P., \& Valtonen, T. (2017). Preparing teacherstudents for twenty-first-century learning practices (PREP 21): a framework for enhancing collaborative problemsolving and strategic learning skills. Teachers and Teaching, 23(1), 25-41. https://doi.org/10.1080/ 13540602.2016.1203772

Järvelä, S., \& Hadwin,A. F. (2013). New frontiers: Regulating learning in CSCL. Educational Psychologist, 48(1), 25-39. https://doi.org/10.1080/00461520.2012.748006

Kim, N. J., Belland, B. R., \&Walker,A.E. (2018). Effectiveness of computer-based scaffolding in the context of problembased learning for STEM education: Bayesian metaanalysis. Educational Psychology Review, 30(2), 397-429. https://doi.org/doi.org/10.1007/s10648-017-94191

Ley Orgánica 1/1990, de 3 de octubre, de Ordenación General del Sistema Educativo. Boletín Oficial del Estado núm. 238, de 4 de octubre de 1990, 28927 a 29842. Recuperado de https://www.boe.es/boe/dias/1990/ 10/04/pdfs/A28927-28942.pdf 
Ley Orgánica 2/2006, de 3 de mayo, de Educación. Boletín Oficial del Estado núm. 106, de 4 de mayo 2006, 17158 a 17207. Recuperado de https: / /www.boe.es/boe/dias/ 2006/05/04/pdfs/A17158-17207.pdf

Ley Orgánica 8/2013, de 9 de diciembre, para la mejora de la calidad Educativa. Boletín Oficial del Estado núm. 295, de 10 de diciembre de 2013, 97858 a 97921 . Recuperado de https://www.boe.es/boe/dias/2013/12/10/ pdfs/BOE-A-2013-12886.pdf

Ley Orgánica 3/2020, de 29 de diciembre, por la que se modifica la Ley Orgánica 2/2006, de 3 de mayo, de Educación. Boletín Oficial del Estado núm. 340 de diciembre, 122868 a 122953. Recuperado de https:// www.boe.es/eli/es/lo/2020/12/29/3/dof/spa/pdf

Lipnevich,A.A., Panadero, E., Gjicali, K., \& Fraile, J. (2021). What's on the syllabus? An analysis of assessment criteria in first year courses across US and Spanish universities. Educational Assessment, Evaluation and Accountability. https: / /doi.org/10.1007/s11092-021-09357-9

López Pastor,V. M. (2017). Evaluación formativa y compartida: evaluar para aprender y la implicación del alumnado en los procesos de evaluación y aprendizaje. EnV.M.LópezPastor \& Á. Pérez-Pueyo (Eds.), Evaluación formativa y compartida en educación: experiencias de éxito en todas las etapas educativas (pp. 34 68). León: Universidad de León.

Navarro-Patón, R., Arufe-Giraldez, V., \& SancosmedSantaballa, E. (2015). Las actividades en el medio natural en la educación física escolar. Formación y actitud del profesorado de Educación Primaria. Retos, 27, 122-126. https: / / doi.org/10.47197/retos.v0i27.34361

Nicol, D., \& Macfarlane-Dick, D. (2006). Formative assessment and self-regulated learning: a model and seven principles of good feedback practice. Studies in Higher Education, 31(2), 199-218. https: / /doi.org/10.1080/ 03075070600572090

Panadero, E., Broadbent, J., Boud, D., \& Lodge, J. M. (2018). Using formative assessment to influence self- and coregulated learning: the role of evaluative judgement. European Journal of Psychology of Education, 34(3), 535-557. https: / / doi.org/10.1007/s10212-018-0407-8

Panadero, E., Jonsson, A., \& Strijbos, J.W. (2016). Scaffolding self-regulated learning through self-assessment and peer assessment: Guidelines for classroom implementation. En D. Laveault \& L. Allal (Eds.), Assessment for learning: Meeting the challenge of implementation (pp. 311-326). Boston: Springer.

Panadero, E., \& Romero, M. (2014). To rubric or not to rubric? The effects of self-assessment in self-regulation, performance and self-efficacy. Assessment in Education:Principles, Policy \& Practice, 21(2), 133-148. https: / / doi.org/ 10.1080/0969594X.2013.877872

Pérez Pueyo, Á. (2015). El estilo actitudinal en Educación
Física: Evolución en los últimos 20 años. Retos, 29, 207215. https: / / doi.org/10.47197/retos.v0i29.38720

Real Decreto 1670/1993, de 24 de septiembre, por el que se establece el título universitario oficial de Licenciado en Ciencias de la Actividad Física y del Deporte y las directrices generales propias de los planes de estudios conducentes a la obtención del mismo. Boletín Oficial del Estado núm. 251, de 20 de octubre de 1993, 29558 a 299559. Recuperado de https: / /www.boe.es/boe/dias/1993/ 10/20/pdfs/A29558-29559.pdf

Real Decreto 1631 / 2006, de 29 de diciembre, por el que se establecen las enseñanzas mínimas correspondientes a la Educación Secundaria Obligatoria. Boletín Oficial del Estado núm. 5, de 5 de enero de 2007. Recuperado de https: / / www.boe.es/buscar/pdf/2007/BOE-A2007-238-consolidado.pdf

Real Decreto 1105 /2014, de 26 de diciembre, por el que se establece el currículo básico de la Educación Secundaria Obligatoria y del Bachillerato. Boletín Oficial del Estado núm. 3 de 3 de enero de 2015, 169 a 546. Recuperado de https: / / www.boe.es/boe/dias/2015/01/03/pdfs/ BOE-A-2015-37.pdf

Resolución del 18 de septiembre de 2018, ̈̈pde la Secretaría General de Universidades, por la que se publica el Acuerdo del Consejo de Universidades de 17 de septiembre de 2018, por el que se establecen recomendaciones para la propuesta por las universidades de memorias de verificación del título oficial de Grado en Ciencias de laActividad Física y del Deporte. Boletín Oficial del Estado núm. 228, de 20 de septiembre de 2018, 91209 a 91217. Recuperado de https://www.boe.es/boe/dias/2018/09/20/ pdfs/BOE-A-2018-12774.pdf

Topping, K. J. (2009). Peer assessment. Theory into Practice, 48(1), 20-27. https://doi.org/10.1080/ 00405840802577569

Wang,A.I., \&Tahir, R. (2020). The effect of using Kahoot! for learning - A literature review 103818. Computers \& Education, 149, 103818. https://doi.org/10.1016/ j.compedu.2020.103818

Winstone, N. E., Nash, R. A., Parker, M., \& Rowntree, J. (2017). Supporting learners' agentic engagement with feedback: A systematic review and a taxonomy of recipience processes. Educational Psychologist, 52(1), 17$37 . \quad$ https://doi.org/10.1080/ 00461520.2016.1207538

Zabala, M., Dalmau, J. M., Viciana, J., \& Gargallo, E. (2003). Modelo de unidad didáctica para Educación Física: un ejemplo integrado de juegos y deportes y actividades en el medio natural como vehículo de iniciación deportiva. Lecturas: Educación Física y Deportes, 64. 